\title{
ICMI Column - The Mathematical Legacy of Jean-Pierre Kahane
}

Julien Barral (Université Paris 13, Villetaneuse, France), Jacques Peyrière (Université Paris-Sud, Orsay, France) and Hervé Queffélec (Université des Sciences et Technologies de Lille, Villeneuve d'Asca, France)

Jean-Pierre Kahane (1926-2017), an alumnus of École Normale Supérieure, was appointed as a professor at Montpellier University just after his $\mathrm{PhD}$ thesis (prepared at CNRS) and at the Faculté des Sciences d'Orsay in 1962, which was then a part of the Sorbonne and later linked to Universite Paris-Sud. He has been the recipient of numerous awards (the enumeration of which would be tedious) and he was a member of the Académie des Sciences de Paris.

His particularly intense and fruitful scientific activity did not prevent him from assuming various positions to the benefit of the community: for instance, he served as the President of Université Paris-Sud, the President of the MIDIST (a Government agency) and the President of the CIEM. Again, it would be tedious to list all the responsibilities he has assumed.

He was fully concerned with the teaching of mathematics. Indeed, he thought of it as intimately linked with research. We refer to the preceding article by Michèle Artigue for an account on this subject. He also had a special interest in the history of mathematics. In particular, he revisited some of Plato's writings.
Jean-Pierre Kahane acted as the advisor for numerous $\mathrm{PhD}$ theses. He was a patient and attentive advisor; he fully respected the personality of his students. He was a talented organiser and coordinator. He is one of the founders of the mathematics department at Orsay. He created and energised a seminar and a team. Due to his international fame, he was able to attract the most world-wise renowned visitors to Orsay.

His first studies deal with the connections between the mean periodic functions of Laurent Schwartz, entire functions and quasi-analyticity. Of course, this echoes the preoccupations of Szolem Mandelbrojt, his PhD advisor. From him, he inherited a taste for Dirichlet series, on which he kept an eye throughout his career.

Soon, he won international renown, as shown by the list of his early collaborators. Nevertheless, in the 1960s, Jean-Pierre Kahane was (in France) a somewhat singular mathematician, or at least he felt so. The preface of his book with Raphael Salem, "Ensembles parfaits et séries trigonométriques", is the best description of his view of mathematics. Here is an excerpt: 
Il y a quelques dizaines d'années, ce livre aurait pu se passer de cette préface, qui est écrite en guise d'apologie. Aujourd'hui, venant à un moment où la plupart des mathématiciens - et les meilleurs - s'intéressent surtout aux questions de structure, il peut paraître suranné et ressembler en quelque sorte à un herbier. Les auteurs se doivent donc d'expliquer que leur propos n'est en aucune façon réactionnaire. Ils savent la beauté des grandes théories modernes, et que leur puissance est irremplaçable, car sans elles on serait souvent condamné (comme l'a dit Lebesgue) à renoncer à la solution de bien des problèmes à énoncés simples posés depuis fort longtemps. Mais ils pensent que, sans ignorer l'architecture qui domine les êtres mathématiques, il est permis de s'intéresser à ces êtres eux-mêmes qui, pour isolés qu'ils puissent paraître, cachent souvent en eux des propriétés, qui, considérées avec attention, posent des problèmes passionnants ...

The objects that this text refers to are generically called thin sets (Kronecker, Sidon, Helson sets, uniqueness sets, etc.), the study of which has been very intensive in his team for more than a decade.

This book was published in 1962, with a second issue in 1994. It is worth noting that such a preface would nowadays be unnecessary; indeed, this view of mathematics, in particular due to Jean-Pierre Kahane, is widespread.

He left a huge volume of work, of which the main topics are:

- Harmonic analysis and Fourier series, in connection with functional analysis and number theory.

- Probabilistic methods in analysis: fine structure of Brownian motion and construction of fractal sets.

- Harmonic analysis methods in analytic number theory.

- Mandelbrot martingales and multiplicative chaos.

Rather than try and describe all of his contributions in this limited space, it is preferable to put the stress on his works that have had the greatest influence. Most of his works are easily accessible via the book "Selected works"(Kendrick press, 2009).

\section{The Kahane-Katznelson-de Leeuw theorem}

If the sequence $\left(c_{n}\right)_{n \in \mathbb{Z}}$ is square summable, there exists a continuous $2 \pi$-periodic function such that, for all $n \in \mathbb{Z}$, $|\widehat{f}(n)| \geq\left|c_{n}\right|$. This brings a definitive conclusion to lots of previous works. The proof mixes probability and combinatorics, a cocktail that he was fond of. Probabilities (the Khintchine inequalities) give $|\widehat{f}(n)| \geq\left|c_{n}\right|$ and $f \in \bigcap_{p<\infty} L^{p}$. The difficulty is to get $f \in L^{\infty}$. This is achieved via the use of combinatorics.

This result teased several followers: Kisliakov showed that this result still holds for the disc and bidisc algebras. The problem seems to still be open in higher dimension; later on, in 1997, Françoise Piquard proved a similar result for infinite matrices.

\section{The Khintchine-Kahane inequalities}

This is a highly non-straightforward extension of Khintchine inequalities to vector random variables. The idea is expressed "à la Kahane" in his book "Some random series of functions":

If the probability that a sum of the type Rademacher be large is small, then the probability it be very large is very small.

More precisely, if $M$ is the supremum of the norms of partial sums of a Rademacher series $\sum r_{n} x_{n}$ (where the $x_{n}$ lie in an arbitrary Banach space) then, for $t>0$ large enough, one has $\mathbb{P}(M>2 t) \leq \mathbb{P}(M>t)^{2}$. It results that the sum $S$ of this series belongs to the Orlicz space $L^{\psi_{1}}$, where $\psi_{1}(x)=\mathrm{e}^{x}-1$. This seems not to be as good as in the scalar case, where the correct Orlicz function is $\psi_{2}(x)=\mathrm{e}^{x^{2}}-1$. But, as Kwapien showed, this result is self-improving so as to give the optimal Orlicz function $\psi_{2}$.

\section{Slow points of Brownian motion $\mathbf{B}(\mathbf{t})$}

This is a long story. One dimensional Brownian motion was known to have, with probability 1 , continuous trajectories, almost uniformly Hölder $1 / 2$ (not $1 / 2$, of course, because of the iterated logarithm law). It was also known (Paley-WienerZygmund) that it is a.s. everywhere non-differentiable and even everywhere non-Hölder $\alpha$ if $\alpha>1 / 2$. Dvoretzky, asked by Kahane about the case $\alpha=1 / 2$, proved in 1963 the following result. Set

$$
L(t):=\limsup _{h \rightarrow 0} \frac{|B(t+h)-B(t)|}{|h|^{1 / 2}} .
$$

Then, a.s. for all $t, L(t)>0$. In 1974, Orey and Taylor proved that $L(t)=\infty$, and a little more, can happen (such points are so-called "fast points"). Shortly afterwards, by using an astonishingly inventive method, Kahane proved that slow points exist, i.e. points at which the Hölder exponent is $1 / 2$. The proof is detailed in the second issue of his book "Some random series of functions".

\section{Solution of the Bateman-Diamond conjecture}

J.-P. Kahane has always been fascinated by the issue, in the job of a mathematician, of the direction in which one should look for an answer? No, and you look for a counterexample. Yes, and you look for a general proof ... In this respect, he often quoted Carleson's theorem (the solution of the Lusin conjecture) on almost everywhere convergence of Fourier series of square-summable functions on the circle. According to him, Carleson rather believed the answer to be negative. But each attempt in this sense had run into a stone wall. Here was Kahane's comment in 2011:

Finally, the walls he ran into nearly built a POSITIVE answer to the Lusin conjecture!

Quite in this spirit, he obtained, in 1997, when over 70, a positive answer to a conjecture of Bateman and Diamond about generalised prime numbers of Beurling. Here are some details. You start from a discrete and multiplicatively independent subset $\mathrm{P}$ of the half-line $(1,+\infty)$ (the generalised prime numbers) and then consider the multiplicative semi-group $N$ generated by $\mathrm{P}$ (the generalised integers), as well as their respective counting functions

$$
P(x)=\operatorname{card} \mathrm{P} \cap[1, x] \text { and } N(x)=\operatorname{card} \mathrm{N} \cap[1, x] .
$$

Beurling showed that by setting $N(x)=D x+x \varepsilon(x)$, with $D$ a positive constant, the assumption $\varepsilon(x)=\mathrm{O}\left((\log x)^{-\alpha}\right)$ for some $\alpha>3 / 2$ is enough to imply the Prime Numbers Theorem (PNT):

$$
P(x) \sim \frac{x}{\log x} .
$$

In this context, the less demanding condition

$$
\int_{1}^{\infty}(\varepsilon(x) \log x)^{2} \frac{d x}{x}<\infty
$$


naturally emerges. Bateman and Diamond asked whether Condition (1) implies the PNT. After failing to build a counterexample and having analysed the reasons of that failure, Kahane was led, in Carleson's style, to a positive answer to the conjecture. The (quite elegant) method uses Fourier analysis and, notably, the non-vanishing of $\zeta$, the zeta function naturally associated with $\mathrm{N}$, on the line $\mathfrak{R} s=1$; this is by interpreting the hypothesis as the membership of $t \mapsto \zeta(1+i t)$ in the Sobolev space $H^{1}$ and making use of the local properties of functions in that space, notably the property

$$
f \in H^{1} \Rightarrow\left|f\left(t_{0}+h\right)-f\left(t_{0}\right)\right|=o\left(|h|^{1 / 2}\right) .
$$

(Surprisingly enough, this is somewhat reminiscent of slow points of Brownian motion.) Jean-Pierre Kahane was particularly proud of this achievement.

\section{The Mandelbrot martingales}

Let $c \geq 2$ be an integer and $W$ a non-negative random variable of expectation 1 . We consider a family $W_{j_{1}, j_{2}, \cdots, j_{n}}$, indexed by the finite sequences of numbers between 1 and $c$, of independent random variables equidistributed with $W$. Each sequence $j_{1}, j_{2}, \cdots, j_{n}$ determines a $c$-adic subinterval $I_{j_{1}, j_{2}, \cdots, j_{n}}$ of $[0,1]$. Let $\mu_{n}$ be the measure whose density with respect to the Lebesgue measure on each interval $I_{j_{1}, j_{2}, \cdots, j_{n}}$ is the constant $W_{j_{1}} W_{j_{1}, j_{2}} \cdots W_{j_{1}, j_{2}, \cdots, j_{n}}$. Its total mass is the random variable

$$
Y_{n}=c^{-n} \sum_{1 \leq j_{1} j_{2} \cdots j_{n} \leq c} W_{j_{1}} W_{j_{1}, j_{2}} \cdots W_{j_{1}, j_{2}, \cdots, j_{n}} .
$$

This is a non-negative martingale so it converges a.s. to a random variable $Y$. Of course, the expectation of $Y$ does not exceed 1. It may happen that $Y=0$ with probability 1 . When the expectation of $Y$ equals 1 , the sequence of measures $\mu_{n}$ has, with probability 1 , a non-trivial weak limit $\mu$. This construction was devised by B. Mandelbrot to statistically describe turbulent fluids - in this case, $\mu$ is interpreted as energy dissipation - or, more generally, to account for intermittency. So, it was crucial to determine whether the expectation of $Y$ differs from 1 and Mandelbrot constructed a few conjectures. These have been answered positively by Kahane. Here are the results.

- For $p>1$, the martingale (2) converges in $L^{p}$ if and only if $\mathbb{E} W^{p}<c^{p-1}(1974)$.

- The martingale converges in $L^{1}$ if and only if $\mathbb{E} W \log W<$ $\log c$ (1976).

- When this martingale does not converge in $L^{1}$, it completely degenerates, i.e. $\mathbb{E} Y=0$.

The 1974 result was already difficult but the one in 1976 is a true feat of strength.

These works had longlasting influence. Other proofs have been devised and lots of generalisations have been studied.

\section{Multiplicative chaos}

In 1974, B. Mandelbrot introduced the previous martingales as a toy model for energy dissipation in fully developed turbulence. The more realistic model he had considered consisted of a basis-free lognormal multiplicative process, which was rigorously founded by Kahane in "Sur le chaos multiplicatif" in 1985, where Gaussian multiplicative chaos theory is elaborated. Then, Kahane unified both models in the more abstract
$T$-martingale setting, of which there is a short presentation here.

One considers $(\Omega, \mathcal{A}, \mathbb{P})$ a probability space, $(T, d)$ a locally compact metric space and

$$
Q=\left(Q_{n}: T \times \Omega \rightarrow \mathbb{R}_{+}\right)_{n \geq 1}
$$

a sequence of measurable maps such that for all $t \in T$, $\left(Q_{n}(t, \cdot)\right)_{n \geq 1}$ is a martingale of expectation 1 .

Given $\sigma$ any Radon measure on $T$, the sequence $\left(Q_{n} \sigma\right)_{n \geq 1}$ vaguely converges almost surely to a Radon measure $Q \sigma$ and, as distributions $\mathbb{E}\left(Q_{n} \sigma\right)$ and $\sigma$ satisfy

$$
\mathbb{E}(Q \sigma) \leq \sigma \quad \text { (by Fatou's Lemma), }
$$

if $Q \sigma \neq 0$ with positive probability, one says that $Q \sigma$ is nondegenerate. Basic questions are then:

1. When is $Q \sigma$ non-degenerate and when does the equality $\mathbb{E}(Q \sigma)=\sigma$ hold? In the case of degeneracy, how can $Q \sigma_{n}$ be renormalised to get a non-trivial limit, at least in law?

2. Suppose $Q \sigma$ is non-degenerate.

- Which moments of the total mass of $Q \sigma$ restricted to compact sets are finite?

- What is the Hausdorff dimension of $Q \sigma$ ?

- What is the multifractal nature of $Q \sigma$, i.e. what are the Hausdorff dimensions of the sets

$$
E(\alpha)=\left\{t \in T: \liminf _{r \rightarrow 0^{+}} \frac{\log (Q \sigma(B(t, r)))}{\log (r)}=\alpha\right\} ?
$$

Gaussian mutiplicative chaos as defined by Kahane corresponds to

$$
Q_{n}(t)=\prod_{k=1}^{n} P_{k}(t), \quad \text { with } P_{n}(t)=\exp \left(X_{n}(t)-\frac{1}{2} \mathbb{E} X_{n}^{2}(t)\right),
$$

where $X_{n}(t)$ are independent Gaussian centered random functions on $T$. The distribution of $P_{n}$ depends only on the correlation function

$$
p_{n}(t, s)=\mathbb{E}\left(X_{n}(t) X_{n}(s)\right)
$$

and the distribution of $Q \sigma$ only depends on

$$
q(t, s)=\sum_{n=1}^{\infty} p_{n}(t, s)
$$

and not on the order of summation if the $p_{n}$ are non-negative. This non-trivial achievement is based on fundamental inequalities that Kahane obtained in this context. Let $\left(X_{i}\right)_{1 \leq i \leq n}$ and $\left(Y_{i}\right)_{1 \leq i \leq n}$ be two centered Gaussian vectors such that

$$
\forall i, j, \quad \mathbb{E}\left(X_{i} X_{j}\right) \leq \mathbb{E}\left(Y_{i} Y_{j}\right) .
$$

Then, for all non-negative weights $\left(p_{i}\right)_{1 \leq i \leq n}$ and all convex (resp. concave) functions $F: \mathbb{R}_{+} \rightarrow \mathbb{R}$, with almost polynomial growth at infinity,

$$
\mathbb{E}\left(F\left(\sum_{i=1}^{n} p_{i} \mathrm{e}^{X_{i}-\frac{1}{2} \mathbb{E}\left(X_{i}^{2}\right)}\right)\right) \leq(\text { resp. } \geq) \mathbb{E}\left(F\left(\sum_{i=1}^{n} p_{i} \mathrm{e}^{Y_{i}-\frac{1}{2} \mathbb{E}\left(Y_{i}^{2}\right)}\right)\right) .
$$

A case of particular interest is when the Gaussian field $\sum_{n=1}^{\infty} X_{n}(t)$ (seen as a distribution) is log-correlated, i.e.

$$
q(t, s)=u \log ^{+} \frac{1}{d(t, s)}+O(1) \quad(u>0) .
$$

Then, if

$$
\sup _{B} N\left(\frac{1}{2} \operatorname{diam}(B), B\right)<\infty,
$$

where $N(\delta, B)$ stands for the covering number of the closed ball $B$ by closed balls of radii $\delta$, Kahane shows that: 
(1) If $\operatorname{dim} T<u / 2$ then for all $\sigma$ one has $Q \sigma=0$.

(2) If $\operatorname{dim} T>u / 2$ then the infimum of the dimensions of random Borel sets on which the non-vanishing measures $Q \sigma$ are concentrated is $\operatorname{dim} T-u / 2$.

Moreover, when $(T, d)$ is the Euclidean space $\mathbb{R}^{d}$ and $\sigma=$ Lebesgue then $\operatorname{dim} T<u / 2$ can be replaced by $\operatorname{dim} T \leq u / 2$ in (1). These results are partly based on Peyrière's approach to the dimensions of limits of Mandelbrot martingales and Kahane's observation of a deep principle of composition for multiplicative chaos.

Questions of non-degeneracy, moments, dimension and multifractal analysis were then solved for various classes of models of $T$-martingales (works by Kahane, Holley and Waymire, Molchan, Falconer, Fan, Barral and Mandelbrot, Bacry and Muzy, Rhodes, Sohier and Vargas, and Barral and Jin), including log-correlated Gaussian multiplicative chaos.

The answer to the renormalisation question came recently for Mandelbrot martingales (in works by Aidekon and Shi, Webb, Madaule, and Barral, Rhodes and Vargas) and for some log-correlated Gaussian multiplicative chaos on $\mathbb{R}^{d}$ (in works by Duplantier, Rhodes, Sheffield and Vargas, and Madaule, Rhodes and Vargas). Both situations led to similarly beautiful results. In the latter case, if we take into account the dependence of $Q_{n}$ in the parameter $u$, if $X_{n} \sim \mathcal{N}(0,1)$ and $\sigma$ is the Lebesgue measure then, in the critical case $u=2 d$, the normalised sequence $\sqrt{n}\left(Q_{u, n} \sigma\right)$ weakly converges in probability to a positive continuous random measure $\mu$ of dimension 0 , while if $u>2 d$ then $n^{\frac{3 \sqrt{u}}{2 \sqrt{2 d}}} \mathrm{e}^{\left(\frac{\sqrt{u}}{\sqrt{2}}-\sqrt{d}\right)^{2} n}\left(Q_{u, n} \sigma\right)$ weakly converges in law to a purely atomic stable random measure, whose atoms are placed according to a Poisson point process with intensity given by $\mu$. Note that complex $T$-martingales and their renormalisation have also been investigated.

Gaussian multiplicative chaos theory was further developed over the last decade with two related motivations: constructing a theory associating random measures to nonnegative kernels $q(t, s)$ of positive type but not necessarily of $\sigma$-positive type (works by Robert and Vargas, Duplantier and Sheffield, Rhodes and Vargas, Shamov and Berestycki); and developing a rigorous probabilistic theory of quantum field theory (works by Duplantier, Miller and Sheffield, David, Kupianen, Rhodes and Vargas, and Aru, Hung and Sun). It turns out that, in certain cases, not only can one characterise the finiteness of moments but one can determine the law of the total mass! (See recent works by Kupianen, Rhodes and Vargas, and Rémy.)

Random coverings, multiplicative chaos and subordinators Multiplicative chaos is related to some random covering problems, which were among the favourites of Kahane. Along with his students Billard and El Hélou, he was among the main protagonists (with Erdös, Hawkes, Mandelbrot and Shepp) in the study and solution of a random covering problem on the circle raised by Dvoretzky in 1956, and of its extension to tori. Kahane also worked on the closely related and more tractable Poisson covering problem in Euclidean spaces introduced by Mandelbrot (1972). Let us discuss the latter model in dimension 1.

Denote the Lebesgue measure on $\mathbb{R}$ by $\lambda$ and fix a positive Borel measure $\mu$ on $(0, \infty)$. Consider a Poisson point pro- cess of intensity $\lambda \otimes \mu$, which is a random discrete subset of $\mathbb{R} \times(0, \infty)$ such that the number of its point in a given Borel set $B$ is a Poisson r.v. of parameter $\lambda \otimes \mu(B)$ and the number of points associated with disjoint Borel sets are independent. Denote the points of this process by $\left(x_{i}, y_{i}\right)$ and associate to them the open intervals $\left(x_{i}, x_{i}+y_{i}\right)$. Let $G=\bigcup_{i}\left(x_{i}, x_{i}+y_{i}\right)$. The problem is to determine whether $G=\mathbb{R}$ a.s. or not. The answer was given by Shepp (1972), simultaneously with the answer to Dvoretzky's problem:

$$
\begin{aligned}
& G=\mathbb{R} \text { a.s. or } G \neq \mathbb{R} \\
& \text { a.s. according to } \int_{0}^{\infty} \mathrm{e}^{-t} k(t) \mathrm{d} t=\infty \text { or not, }
\end{aligned}
$$

where

$$
k(t)=\exp \int_{t}^{\infty} \mu(y, \infty) \mathrm{d} y .
$$

Kahane revisits this result in the late 1980s. He gives a new proof based on a stopping time idea due to Janson and also uses a potential theory approach to get the following finer result. If $K$ is a compact subset of $\mathbb{R}$ then $K \subset G$ a.s. or $K \notin \bigcap_{n \geq 1} \bigcup_{i: y_{i}<1 / n}\left(x_{i}, x_{i}+y_{i}\right)$ a.s. according to whether $\operatorname{Cap}_{k}(K)=0$ or not. Multiplicative chaos comes into play when $\operatorname{Cap}_{k}(K)>0$, i.e. there exists a Borel probability measure $\sigma$ on $K$ such that

$$
\int_{K^{2}} k(t-s) \mathrm{d} \sigma(t) \mathrm{d} \sigma(s)<\infty .
$$

Indeed, for $n \geq 1$, setting $G_{n}=\bigcup_{i: y_{i} \geq 1 / n}\left(x_{i}, x_{i}+y_{i}\right)$ and

$$
Q_{n}(t)=\frac{1-\mathbf{1}_{G_{n}}(t)}{1-\mathbb{P}\left(t \in G_{n}\right)},
$$

(4) is equivalent to $L^{2}$-convergence of the $K$-martingale $\left(Q_{n} \sigma\right)(K)$.

Now, consider $G_{+}=\bigcup_{i: x_{i}>0}\left(x_{i}, x_{i}+y_{i}\right)$. In the same period, Kahane shows that when

$$
\int_{0}^{1} \exp \left(\int_{x}^{1} \mu(y, \infty) \mathrm{d} y\right) \mathrm{d} x<\infty,
$$

there exists some Radon measure $\sigma$ supported on $\mathbb{R}_{+}$such that $Q \sigma$ is supported on $\mathbb{R}_{+} \backslash G_{+}$, the set of uncovered points, and $Q \sigma$ is the image of the Lebesgue measure by some Lévy subordinator. This implies a result by Fitzsimmons, Firstedt and Shepp (partially proved by Mandelbrot), claiming that, in this case, $\mathbb{R}_{+} \backslash G_{+}$is the closure of the range of this subordinator but provides a new interpretation in terms of multiplicative chaos. In particular, an interesting consequence of this approach is that local times of Brownian motion are the indefinite integrals of limits of $\mathbb{R}$-martingales. It should be noted that the topic of random coverings is currently particularly active.

J.-P. Kahane's influence turned out to be amazing. Firstly, he was always ready to discuss and talk about mathematics. We remember J. P. Serre asking him: "We were wondering if we could get from you a talk on Brownian motion next week?" He instantly replied: "I agree." And he delivered several talks at the Bourbaki seminar, notably on Beurling's works, to whom he first asked for agreement. Facing a lack of reply from the latter, he sent a message: "In three days, I present your work at the Bourbaki seminar," no longer asking for agreement. He then received agreement with an abrupt 
“OK”. Much more recently (in 2014), J. P. Kahane delivered a talk on Brownian motion, white noise and Langevin's equation at the "BNF" (Bibliothèque Nationale de France), in front of a large audience comprised of many teenagers. His aptitude for "doing mathematics" and writing equations, while remaining sufficiently general to avoid the young listeners getting lost, was impressive.

J.-P. Kahane wrote four advanced books:

- "Ensembles parfaits et Séries Trigonométriques" with R. Salem (1963); second edition in 1994. This book has not aged. In particular, it provides a clear account on capacities and Hausdorff dimension.

- "Séries de Fourier absolument convergentes" (1970). This book contains magnificent applications of Banach algebras (Shilov idempotents theorem) to the zero sets of analytic functions in the Wiener class.

- "Some random series of functions" (1968, reissued in 1985). This book is still an obligatory reference.

- "Séries de Fourier et Ondelettes" with P. G. Lemarié (1998, reissued in 2016).

This last work shows the taste and ability of J.-P. Kahane for history, focusing on the development of ideas rather than on persons. Before the wavelet part, written by P.-G. Lemarié, he writes about the contributions of the great precursors: Euler, Fourier, Dirichlet, Weierstrass, Cantor, Riemann and Lebesgue. His analysis of their ideas and achievements is very exciting.

Jean-Pierre Kahane will remain an example thanks to his enthusiasm, his quest for invention and his preoccupation to explain and transmit his knowledge and experience. His influence will continue for a long time.

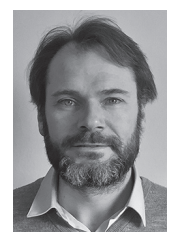

Julien Barral [barral@math.univ-paris13.fr] is a Professor of Mathematics at Paris 13 University. He is Chairman of Laboratoire d'Analyse, Géométrie et applications (CNRS UMR 7539). He graduated from Paris-Sud University, where he got his PhD under Jacques Peyrière supervision. He works in probability, ergodic theory and fractal geometry, with a special interest to multifractals.

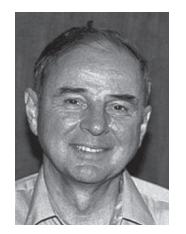

Jacques Peyrière [jacques.peyriere@math. u-psud.fr] is an Emeritus Professor of Mathematics at Paris Sud University (Orsay). His research interests are Harmonic Analysis, Probability Theory, and Fractals. He is a former student of Jean-Pierre Kahane.

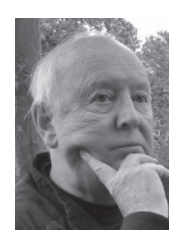

Hervé Queffélec_[herve.queffelec@ univ-lille1.fr] is now an Emeritus Professor at the University of Lille, after having been a "maître de conferences" at the University of Orsay till 1992 and a Professor at the University of Lille 1 from 1992 to 2011.

His research concerns are harmonic analysis in connection with analytic number theory and Dirichlet series, as well as Banach spaces of analytic functions and their operators. He is a former student of Jean-Pierre Kahane. 